\title{
Lócus de Controle, Personalidade, Autoeficácia e Aspectos Emocionais em Aspirantes A Piloto
}

\author{
Manuela Polidoro Lima \\ Luiza Signorelli \\ Maria Laura Del Huerto \\ Pontifícia Universidade Católica do Rio Grande do Sul - PUCRS, Porto Alegre-RS, Brasil \\ Thiago Gomes de Castro \\ Universidade Federal do Rio Grande do Sul - UFRGS, Porto Alegre-RS, Brasil \\ Manoela Ziebell de Oliveira \\ Tatiana Quarti Irigaray \\ Pontifícia Universidade Católica do Rio Grande do Sul - PUCRS, Porto Alegre-RS, Brasil
}

\section{Resumo}

O principal objetivo deste estudo foi verificar a relação entre lócus de controle (LOC), fatores de personalidade, autoeficácia, sintomas de depressão, ansiedade e estresse em estudantes de graduação em Ciências Aeronáuticas. 109 participantes foram avaliados utilizando: Inventário dos Cinco Grandes Fatores da Personalidade (IGFP-5); Depression, Anxiety and Stress Scale - DASS-21; Escala de Autoeficácia Geral Percebida (EAGP), e Escala de Lócus de Controle para Segurança em Aviação. Os resultados indicaram que: Conscienciosidade correlacionou-se com autoeficácia e aspectos emocionais; Abertura e Extroversão obtiveram correlação com autoeficácia; Neuroticismo obteve correlação com autoeficácia e aspectos emocionais. Compreende-se a relevância da autoeficácia em estudantes e profissionais da aeronáutica, uma vez que tal construto relaciona-se com a saúde mental e com os comportamentos de risco.

Palavras-chave: Autoeficácia, controle interno-externo, ansiedade, depressão, estresse psicológico

Abstract: Locus of Control, Personality, Self-Efficacy And Emotional Aspects Of Pilot Students

This study was conducted to verify the relationship between locus of control (LOC), personality characteristics, self-efficacy, and depression, anxiety, and stress symptoms in adults enrolled in an Aeronautical Science graduation course. One hundred and nine participants were assessed using the following: Big Five Inventory (IGFP-5); Depression, Anxiety and Stress Scale - DASS-21; General Perceived Self-Efficacy Scale (GPSS); and Aviation Safety Locus of Control Scale. Results indicated that: Conscientiousness is correlated to self-efficacy and emotional aspects; Openness and Extraversion are correlated to self-efficacy; and Neuroticism is correlated to self-efficacy and emotional aspects. These results suggest the relevance of self-efficacy in aeronautical students and professionals, since such construct is related to both mental health and rick behavior.

Keywords: Self-efficacy, locus of control, anxiety, depression, stress

Resumen: Lócus de Control, Personalidad, Autoeficacia Y Aspectos Emocionales En Aspirantes A Piloto

El principal objetivo de este estudio fue comprobar la relación entre locus de control (LOC), factores de personalidad, autoeficacia, síntomas de depresión, ansiedad y estrés en estudiantes de graduación en Ciencias Aeronáuticas. Fueron evaluados 109 participantes, utilizando: Inventario de los Cinco Grandes Factores de la Personalidad (IGFP); Depression, Anxiety and Stress Scale - DASS-21; Escala de Autoeficacia General Percibida (EAGP), y Escala de Locus de control para seguridad en aviación. Los resultados indicaron que: Responsabilidad se correlacionó con autoeficacia y aspectos emocionales; Apertura y Extraversión obtuvieron correlación con autoeficacia; Neuroticismo obtuvo correlación con autoeficacia y aspectos emocionales. Se comprende la relevancia de la autoeficacia en estudiantes y profesionales de la aeronáutica, ya que tal constructo se relaciona con la salud mental y con los comportamientos de riesgo.

Palabras clave: Autoeficacia, locus de control, ansiedad, depresión, estrés

\footnotetext{
${ }^{1}$ Endereço para correspondência: Rua Vicente da Fontoura, 2954, 90640-003, Rio Branco, Porto Alegre, RS. E-mail: manuela.lima@acad.pucrs.br
} 
Os primeiros estudos sobre personalidade em pilotos tiveram início no meio militar com o objetivo de identificar pilotos considerados aptos ao programa de treinamento de voo, antes de iniciar tal treinamento. Essa estratégia auxiliava na exclusão de pilotos com características de personalidade inadequadas para a função e evitava despesas na formação de profissionais com altas chances de falhar durante a preparação para a atuação como profissional (Ganesh \& Joseph, 2005).

Os estudos na área da segurança em aviação têm apontado que o desempenho do piloto depende de fatores de personalidade, que podem influenciar diretamente sua maneira de reagir perante situações típicas de trabalho, além de contribuir para a ocorrência de acidentes e incidentes aeronáuticos (Ganesh \& Joseph, 2005; Dolgin, Lambirth, Rentmeister-Bryant, \& Moore, 2003). Os fatores de personalidade podem ainda impactar as relações interpessoais e o trabalho em equipe, manifestando-se na gestão da tripulação aérea (Ganesh \& Joseph, 2005).

Além da personalidade, outro fator humano que vêm sendo investigado na literatura de segurança em aviação é a percepção de risco e lócus de controle entre pilotos (You, Ji, \& Han, 2013). Lócus de controle (LOC) é um constructo que indica o quanto o sujeito acredita ter o controle sob os fatores que influenciam os desfechos em sua vida (Shell \& Husman, 2001). Essa percepção influencia o bom funcionamento do indivíduo em termos de saúde física e mental, pois representa uma espécie de reserva cognitiva da qual o sujeito dispõe e que orienta suas ações (Heckhausen, Wrosch, \& Schulz, 2010).

A percepção de controle está ligada às crenças que o indivíduo tem em relação a sua influência e responsabilidade diante das circunstâncias da vida (Infurna \& Okun, 2015). Estudos indicam que, quanto mais o sujeito atribui a responsabilidade sobre a própria vida a si mesmo, menores são as chances de ele se colocar em risco, ou seja, mais protegido ele está da mortalidade ao longo do tempo (Infurna, Ram, \& Gerstorf, 2013).

O LOC é um constructo que pode ter dois tipos diferentes de orientação: interna ou externa. Culturalmente, quando um evento não é completamente condizente com a ação intencional das pessoas, ele é atribuído a causas externas, como a sorte, o destino ou o poder de outros (Galindo \& Ardila, 2012). O indivíduo que possui maior LOC externo considera, portanto, que os fatores que influenciam sua vida são externos a ele, ou seja, fogem ao seu controle. Por outro lado, aquele que possui um LOC interno elevado se percebe responsável pelos desfechos da própria vida, os quais dependem principalmente de sua capacidade e esforço (You et al., 2013).
Estudos mostram que o LOC influencia o bom desempenho das pessoas ao longo da vida, justamente por estar ligado de forma tão íntima às crenças em relação ao próprio potencial (Ruthig et al., 2008). Tais crenças influenciarão as perspectivas de futuro, posto que estão relacionadas à motivação para a mudança e à autoeficácia, que é definida pelas crenças que a pessoa tem em relação a suas habilidades ou recursos para realizar determinada tarefa (Shell \& Husman, 2001.

A autoeficácia pode exercer também uma função mediadora, pois permite que os traços de personalidade do indivíduo sejam manifestos por meio do comportamento (Caprara, Vecchione, Alessandri, Gerbino, \& Barbaranelli, 2011). Além disso, essa variável influencia diretamente a dimensão predominante do LOC. Assim, quanto maior a medida de LOC interno, maior também a autoeficácia do sujeito, e quanto maior a medida de LOC externo, menor a autoeficácia (Baptista, Alves, \& Santos, 2008).

A construção de LOC tem sido aplicada para compreender a depressão e as respostas das pessoas a eventos estressantes do cotidiano (Lazarus \& Folkman, 1984). Um cenário em que estudos dessa natureza se mostra extremamente pertinente é o da aviação. $\mathrm{O}$ contexto de trabalho de profissionais da aviação é considerado um dos mais estressantes (Widyahening, 2007), pois a atividade do piloto requer clareza e precisão na tomada de decisão, além de rapidez para acompanhar a velocidade em que funcionam os sistemas e equipamentos (Itani, 2009). Esses aspectos podem gerar excessivos níveis de estresse e fadiga da tripulação de voo (Bennet, 2003).

Os pilotos da aviação civil, em geral, encontram-se submetidos a situações de alta demanda física e psicológica, como horas concentradas de atividade durante jornadas irregulares de trabalho e repouso, longe de sua casa e de seus familiares, muitas vezes em fusos horários diferentes (Itani, 2009). Além disso, estão submetidos a situações de alta demanda psicológica e baixo controle sobre seu trabalho, devido às mudanças operacionais e às circunstâncias ambientais inerentes à função (Causse, Dehais, \& Pastor, 2011). Todos estes fatores podem facilitar o surgimento de transtornos mentais comuns, representando um risco potencial à segurança de voo (Feijó, Câmara, \& Luiz, 2014).

Estudos desenvolvidos com pilotos encontraram prevalências maiores que 39\% de transtornos mentais comuns entre profissionais dessa área (Feijó et al., 2014; Widyahening, 2007). Adicionalmente, apontam forte associação entre alta exigência por desempenho, feita pelas companhias aéreas, e prevalência de transtornos mentais, além de identificar estresse no trabalho e tensões familiares como fatores de risco para a saúde mental dos pilotos. 
Após esta explanação, percebe-se que tanto a função do piloto quanto seu ambiente de trabalho, envolvem altos níveis de responsabilidade e estresse, e reduzida possibilidade de controle. Neste cenário, fatores de personalidade dos profissionais desta área e a presença de psicopatologias podem ser decisivos para a qualidade do desempenho da função (Ganesh \& Joseph, 2005; Dolgin et al., 2003). Esses aspectos podem estar também associados ao grau de mobilização de recursos cognitivos para realizar com sucesso as tarefas exigidas, bem como o quanto esses profissionais acreditam que os acontecimentos em sua vida são controlados por eles mesmos ou por fontes externas. Portanto, este estudo tem como principal problema de pesquisa: o lócus de controle (LOC) associa-se com características de personalidade, autoeficácia, sintomas depressivos, de ansiedade e de estresse em estudantes de Ciências Aeronáuticas?

Assim, acredita-se que conhecer tais características nesta população pode ser de extrema relevância para a compreensão de fatores que interferem direta ou indiretamente no trabalho, como: relações interpessoais na cabine, relação homem-máquina e LOC sobre a tomada de decisão em situações estressantes (Green, Muir, James, Gradwell, \& Green, 1996). Dessa forma, o objetivo do presente estudo foi verificar a relação entre LOC, características de personalidade, autoeficácia, sintomas depressivos, de ansiedade e de estresse em adultos matriculados em um curso de graduação em Ciências Aeronáuticas. Ainda, verificou-se quais as variáveis preditoras para o LOC nessa população.

\section{Método}

\section{Participantes}

A amostra foi composta por 109 adultos com idades entre 17 e 30 anos $(M=21,03 ; D P=2,73)$, estudantes de um curso de Ciências Aeronáuticas, que voluntariamente optaram por participar do estudo. Participantes que preencheram parcialmente os instrumentos não foram incluídos nas análises.

\section{Instrumentos}

Os participantes responderam um questionário online constituído de uma ficha de caracterização dos participantes, além de escalas para avaliar as variáveis de interesse deste estudo. Os instrumentos encontram-se descritos a seguir:

Ficha de Dados Sociodemográficos: inclui as variáveis idade, sexo, estado civil, semestre do curso, situação de moradia, estado de saúde física e mental, uso de medicação, uso de cigarro e bebida (quantidade e frequência).

Inventário dos Cinco Grandes Fatores da Personalidade (IGFP-5 - Benet-Martínez \& John, 1998): composto por 44 itens, estruturados em sentenças simples e respondidos em uma escala de respostas do tipo Likert de cinco pontos. $\mathrm{O}$ instrumento foi adaptado e validado por Andrade (2008) para o contexto brasileiro, considerando os seguintes índices de alfa de Cronbach: fator de personalidade Abertura à experiência 0,65 ; fator Neuroticismo 0,75; fator Extroversão 0,75; Conscienciosidade 0,64 e fator de personalidade amabilidade 0,69.

Depression, Anxiety and Stress Scale - DASS-21 (Lovibond \& Lovibond, 1995): trata-se de uma escala composta por 21 itens, que se destina avaliar sintomas de depressão, ansiedade e estresse. Cada item consiste em uma afirmação acerca de sintomas emocionais negativos, e o participante deve responder de acordo com uma escala de respostas do tipo Likert, marcando o quanto cada enunciado se aplicou a ele durante a última semana.

Escala de Autoeficácia Geral Percebida (EAGP) (General Perceived Self-Efficacy Scale - GPSS; Schwarzer \& Jerusalem, 1995): trata-se de uma escala de autorrelato com 10 itens, que avalia a autoeficácia percebida. Os itens da escala são respondidos em formato Likert, e a soma das respostas gera um escore total, assim, quanto maior o escore, maior o nível de autoeficácia.

Escala de Lócus de Controle para Segurança em Aviação (Hunter, 2002): composta por 20 itens, subdividida em dois fatores, LOC externo (E) e LOC interno (I). As 20 afirmativas apresentam a possibilidade de resposta em uma escala de cinco pontos tipo Likert de 1 a 5, sendo 1 concordo fortemente e 5 discordo fortemente. Os itens versam sobre situações cotidianas de pilotos nas quais devem afirmar o quanto se percebem no controle ou falta de controle sobre situações de aviação. $\mathrm{O}$ instrumento será adaptado e validado para pilotos brasileiros no presente estudo.

\section{Procedimentos}

A coleta de dados foi realizada de maneira online, por meio de um questionário eletrônico construído pela plataforma de pesquisa Google Forms. O Termo de Consentimento Livre e Esclarecido foi aplicado aos participantes do estudo e lhes foram garantidos sigilo e anonimato, sendo sua participação voluntária. O projeto foi enviado e aprovado pela Comissão Científica e pelo Comitê de Ética da Universidade de vinculação dos pesquisadores (CAAE 47545715.0.0000.5336). 


\section{Análise de dados}

Utilizaram-se análises descritivas de média, desvio-padrão e percentual. Correlações entre os fatores de personalidade (IGFP-5), LOC, autoeficácia, sintomas depressivos, de ansiedade e de estresse (DASS-21) foram investigadas por meio de análise de correlação de Pearson. As variáveis que apresentaram correlações significativas com o LOC interno e externo foram incluídas em modelos de regressão linear múltipla com método stepwise. A distribuição dos dados foi verificada por meio do teste de
Kolmogorov-Smirnov. Os dados foram analisados no pacote estatístico SPSS 22 para Windows, considerando-se resultados significativos se $p \leq 0,05$.

\section{Resultados}

A Tabela 1 apresenta a distribuição dos participantes em relação a sexo, estado civil, nível socioeconômico e unidade federativa de procedência. A maioria dos participantes é do sexo masculino, solteira e procedente do sul do Brasil.

Tabela 1

Distribuição conforme sexo, estado civil, nível socioeconômico e unidade federativa

\begin{tabular}{|c|c|c|}
\hline & $n$ & $\%$ \\
\hline \multicolumn{3}{|l|}{ Sexo } \\
\hline Masculino & 100 & 8,30 \\
\hline Feminino & 9 & 91,7 \\
\hline \multicolumn{3}{|l|}{ Estado civil } \\
\hline Solteiro & 106 & 97,2 \\
\hline Casado & 3 & 2,80 \\
\hline \multicolumn{3}{|l|}{ Nível socioeconômico } \\
\hline A & 32 & 29,4 \\
\hline $\mathrm{B}$ & 52 & 47,7 \\
\hline $\mathrm{C}$ & 25 & 22,9 \\
\hline \multicolumn{3}{|l|}{ Unidade federativa } \\
\hline Amazonas & 1 & 0,90 \\
\hline Bahia & 2 & 1,80 \\
\hline Distrito Federal & 2 & 1,80 \\
\hline Espírito Santo & 3 & 2,80 \\
\hline Mato Grosso & 1 & 0,90 \\
\hline Mato Grosso do Sul & 1 & 0,90 \\
\hline Minas Gerais & 2 & 1,80 \\
\hline Paraná & 2 & 1,80 \\
\hline Rio de Janeiro & 2 & 1,80 \\
\hline Rio Grande do Sul & 82 & 75,2 \\
\hline Santa Catarina & 5 & 4,60 \\
\hline São Paulo & 5 & 4,60 \\
\hline Sergipe & 1 & 0,90 \\
\hline
\end{tabular}

Nota. $n=$ amostra; $\%=$ porcentagem

As correlações entre LOC, fatores de personalidade, autoeficácia e sintomas depressivos, de ansiedade e de estresse encontram-se na Tabela 2. A Escala de Autoeficácia obteve correlações significativas e moderadas com todos os fatores de personalidade avaliados pela IGFP-5, exceto com Amabilidade. Apenas uma correlação negativa foi encontrada entre autoeficácia e neuroticismo, enquanto as demais foram positivas. Especificamente nas escalas da DASS-21, o escore em Depressão correlacionou-se positivamente com os fatores de personalidade Neuroticismo e Amabilidade e negativamente com Conscienciosidade. Já os escores em Ansiedade e Estresse tiveram relações significativas com todos os fatores de personalidade, exceto Amabilidade, sendo encontradas correlações negativas 
com os fatores Abertura à Experiência, Extroversão e Conscienciosidade, mas positivas com Neuroticismo. Finalmente, o escore de LOC Interno relacionou-se positivamente apenas com o fator de personalidade Conscienciosidade.
Na Tabela 3 constam os modelos explicativos para o LOC. Os resultados indicaram que a autoeficácia foi a única variável preditora significativa tanto para o LOC interno quanto externo, explicando, respectivamente, $6,8 \%$ e $8,3 \%$ da variabilidade.

Tabela 2

Correlação entre os Escores Locus de Controle, IGFP-5, DASS-21 e Autoeficácia

\begin{tabular}{|c|c|c|c|c|c|c|c|c|c|c|}
\hline & \multicolumn{2}{|c|}{ LOC } & \multicolumn{5}{|c|}{ IGFP-5 } & \multicolumn{3}{|c|}{ DASS-21 } \\
\hline & 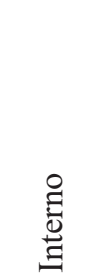 & & 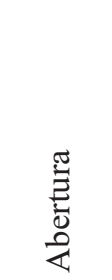 & 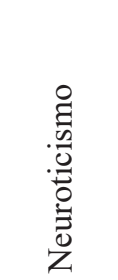 & 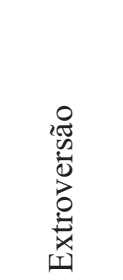 & $\begin{array}{l}\frac{0}{0} \\
\frac{\tilde{J}}{0} \\
\cdot \frac{0}{0} \\
\frac{0}{0} \\
\cdot \frac{0}{0} \\
\tilde{0}\end{array}$ & 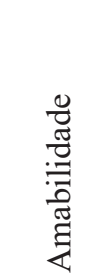 & 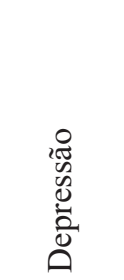 & $\begin{array}{l}\frac{0}{0} \\
\frac{\pi}{0} \\
\frac{d}{2} \\
\frac{Z}{4}\end{array}$ & 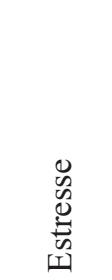 \\
\hline LOC Interno & - & & & & & & & & & \\
\hline LOC Externo & $-0,21^{*}$ & - & & & & & & & & \\
\hline IGFP-5 Abertura & 0,06 & $-0,09$ & - & & & & & & & \\
\hline IGFP-5 Neuroticismo & $-0,12$ & 0,01 & $-0,16$ & - & & & & & & \\
\hline IGFP- 5 Extroversão & 0,09 & $-0,05$ & $0,31^{* * *}$ & $-0,24 * *$ & - & & & & & \\
\hline IGFP-5 Conscienciosidade & $0,26^{* *}$ & $-0,05$ & 0,17 & $-0,23^{*}$ & $0,26^{* *}$ & - & & & & \\
\hline IGFP-5 Amabilidade & 0,06 & $-0,12$ & $0,25^{* *}$ & $0,19 *$ & $0,28^{* *}$ & $-0,07$ & - & & & \\
\hline DASS-21 Depressão & 0,01 & $-0,00$ & $-0,05$ & $0,56^{* * *}$ & $-0,11$ & $-0,29 * *$ & $0,32 * * *$ & - & & \\
\hline DASS-21 Ansiedade & $-0,07$ & 0,05 & $-0,24^{* *}$ & $0,54^{* * *}$ & $-0,33 * * *$ & $-0,36^{* * *}$ & 0,10 & $0,74^{* * *}$ & - & \\
\hline DASS-21 Estresse & $-0,05$ & 0,00 & $-0,27^{* *}$ & $0,57^{* * *}$ & $-0,28^{* *}$ & $-0,19^{*}$ & 0,17 & $0,78^{* * *}$ & $0,79 * * *$ & - \\
\hline Autoeficácia & $0,28^{* *}$ & $-0,30 * * *$ & $0,33^{* * *}$ & $-0,35^{\text {*** }}$ & $0,36^{* * *}$ & $0,44^{* * *}$ & 0,12 & $-0,33^{* * *}$ & $-0,36^{* * *}$ & $-0,32^{* * *}$ \\
\hline
\end{tabular}

Nota. LOC = Locus de controle; IGFP-5 = Inventário dos Cinco Grandes Fatores de Personalidade; DASS-21 = Escala de Depressão, Ansiedade e Estresse; ${ }^{*} p \leq 0,05 ; * * p \leq 0,01 ;{ }^{* * *} p \leq 0,001$

Tabela 3

Modelos de Regressão Linear Múltipla: Lócus de Controle Interno e Externo

\begin{tabular}{lccccccccc}
\hline \multicolumn{1}{c}{ Modelos } & $B$ & $S E$ & $\beta$ & $t$ & IC 95\% & $R$ & $R^{2} a$ & $F$ & $p$ \\
\hline Locus de controle interno & & & & & & & & & \\
$\quad$ Constante & 24,086 & 3,903 & & 6,170 & $16,348-31,824$ & 0,278 & 0,068 & 8,942 & 0,003 \\
Autoeficácia & 0,350 & 0,117 & 0,278 & 2,990 & $0,118-0,583$ & & & & \\
& & & & & & & & & \\
Locus de controle externo & & & & & & & & \\
$\quad$ Constante & 29,573 & 3,273 & & 9,036 & $23,085-36,061$ & 0,303 & 0,083 & 10,800 & $\leq 0,001$ \\
$\quad$ Autoeficácia & $-0,323$ & 0,098 & $-0,303$ & $-3,286$ & $-0,518--0,128$ & & & & \\
\hline
\end{tabular}

\section{Discussão}

O presente estudo buscou verificar a relação entre lócus de controle, características de personalidade, autoeficácia, sintomas depressivos, de ansiedade e de estresse em adultos graduandos do curso de Ciências Aeronáuticas. Os resultados apontaram que o LOC de orientação interna associou-se positivamente com o fator de personalidade conscienciosidade. Dentre os cinco fatores de personalidade estudados, a conscienciosidade apresenta-se como o preditor mais consistente e válido de desempenho dentro do contexto das organizações (Hurtz \& Donovan, 2000). Da mesma forma, o LOC - considerado, por alguns estudiosos, uma importante subdimensão da personalidade - também tem sido identificado como preditor potencialmente válido de desempenho no trabalho (Mali, 2013). 
Um estudo desenvolvido por Hunter (2002) encontrou que pilotos com menor LOC interno apresentaram maior tendência a experimentar eventos mais perigosos relacionados à aviação, em comparação a pilotos com LOC interno dominante. Outro estudo sugeriu, de forma complementar, que uma orientação de LOC mais externa está relacionada com a falta de cautela, e à insegurança no trabalho (Pienaar \& De Witte, 2016), o que pode resultar em falhas ao tomar medidas de precaução para evitar a ocorrência de desfechos desfavoráveis. Dessa forma, indivíduos com alta conscienciosidade - que mantém maior grau de cautela e controle para atingir e lutar por seus objetivos - apresentariam também maior LOC interno.

A autoeficácia associou-se positivamente com os fatores de personalidade Conscienciosidade, Extroversão e Abertura; e negativamente com Neuroticismo. A autoeficácia pode ser vista como um fator que contribui para o desenvolvimento e regulação dos comportamentos que podem ser caracterizados como traços de personalidade (Bandura, 2012). Estes traços parecem exercer papel importante no processo de formação de crenças de autoeficácia (Lent, Brown, \& Hackett, 1994).

A relação encontrada neste estudo entre autoeficácia, extroversão e conscienciosidade parece ser bem fundamentada na literatura (Hartman \& Betz, 2007). Por exemplo, autores indicam que conscienciosidade e autoeficácia podem ser determinantes pessoais decisivos do desempenho acadêmico e profissional (Caprara et al., 2011; Poropat, 2009). Adicionalmente, pessoas com alto nível desse fator de personalidade são consideradas mais organizadas, persistentes, motivadas, trabalhadoras, pontuais e ambiciosas (Noronha, Mansão, \& Nunes, 2012). É possível que essa característica contribua, portanto, para um melhor desempenho nas atividades realizadas e maior autoeficácia. Especialmente quando se considera que um indivíduo com alto senso de autoeficácia é capaz de definir seus objetivos e executar comportamentos eficazes, engajando-se em tarefas de busca de informação e planejamento de ações futuras (Lent et al., 1994).

Ainda em relação à conscienciosidade, Judge e Ilies (2002) observaram correlações positivas com três tipos de motivação para execução de tarefas, sendo uma delas a autoeficácia. Mais especificamente, indivíduos com pontuação alta neste fator demonstraram tendência a se envolverem mais e trabalharem melhor para alcançar seus objetivos (Bandura, 1994), o que poderia levá-los a maiores níveis de autoeficácia e melhor desempenho.

A relação entre Extroversão e autoeficácia, de acordo com De Raad e Schouwenburg (1996), pode ser ambivalente, considerando que a autoeficácia pode interferir no desempenho acadêmico. Extroversão pode exercer um efeito positivo quando se relaciona ao entusiasmo, alto nível de energia e desejo de aprender (Poropat, 2009), o que pode ser um constructo motivacional, o qual melhora o desempenho dos indivíduos em tarefas e atividades. No entanto, indivíduos extrovertidos tendem a ser mais falantes e sociáveis, a considerar-se mais alegres, a apresentar maior motivação e satisfação com a vida, e a se interessar por atividades mais sociáveis e excitantes, capazes de desencadear emoção. Essas características sugerem que possam preferir participar de atividades sociais ao invés de atividades de concentração, esforço e estudo (Ambiel, Noronha, \& Nunes, 2012; Nunes, Hutz, \& Giacomoni, 2009), o que diminuiria sua autoeficácia nas atividades desempenhadas.

A relação entre abertura e autoeficácia também parece ser contraditória na literatura. A relação encontrada neste estudo pode ser justificada pelo fato de que pessoas com alto nível de Abertura tendem a ser mais curiosas, criativas e questionadoras, além de apresentarem maior necessidade de ter experiências variadas (Howard \& Howard, 1995). No entanto, uma pesquisa realizada com estudantes de academias militares indicou que a abertura à experiência teve correlação negativa e fraca com o desempenho militar dos participantes, mas não com o acadêmico (Fosse, Buch, Säfvenbom, \& Martinussen, 2015). Adicionalmente, não apresentou correlação com autoeficácia, resultado que difere do encontrado no presente estudo. Em relação ao fator de personalidade neuroticismo, a relação encontrada indica que sujeitos com maior neuroticismo apresentam menores índices de autoeficácia, associação já apontada por outros estudos (Wang et al., 2014).

Os sintomas depressivos associaram-se positivamente com Amabilidade/ socialização. Estudos prévios demonstraram que este fator de personalidade está associado positivamente com transtornos depressivos (Koorevaar et al., 2013; Wang et al., 2014). Resultado complementar foi observado no estudo realizado por Kim et al. (2016), o qual encontrou que socialização/ amabilidade foi um dos fatores que exerceu efeito sobre sintomas de depressão. Segundo os autores, tanto neuroticismo quanto socialização/amabilidade associaram-se direta e indiretamente com sintomas depressivos. A explicação para esses resultados poderia residir no fato de pessoas com altos índices de socialização/amabilidade serem, em geral, mais generosas, preocupadas e disponíveis para ajudar os outros (Nunes \& Hutz, 2007), o que justificaria o maior risco de desenvolvimento de sintomas depressivos, especialmente quando essas características sofrem influência da perda de alguém ou do rompimento de vínculos afetivos. 
Também pode-se pensar que, justamente por esse fator englobar comportamentos socialmente positivos e a qualidade dos padrões estabelecidos nos relacionamentos (Nunes, Hutz, \& Nunes, 2010), indivíduos que se preocupam muito com outros podem absorver seus problemas com grande intensidade, ao ponto de se sentirem responsáveis por eles, deixando de viver sua própria vida em despeito a estarem sempre disponíveis aos outros e as suas queixas; o que, consequentemente, pode interferir em sua saúde emocional. Apesar dos resultados obtidos neste e em outros estudos, cabe notar que as evidências sobre a relação entre socialização/amabilidade e depressão são ainda inconsistentes (Koorevaar et al., 2013; Roesch, Aldridge, Vickers \& Helvig, 2009).

Os sintomas de depressão, ansiedade e estresse associaram-se positivamente com os fatores de personalidade Neuroticismo e negativamente com Conscienciosidade. Estudos anteriores demonstraram que características de personalidade, como elevados níveis de neuroticismo, por exemplo, estão associadas positivamente com o diagnóstico e prognóstico de transtornos depressivos (Koorevaar et al., 2013; Wang et al., 2014). Complementarmente, Karsten, Penninx, Riese, Ormel, Nolen, e Hartman, (2012) encontraram que alguns fatores de personalidade são preditores do desenvolvimento de transtornos afetivos, tanto depressivos quanto ansiosos, e um deles é o neuroticismo. Este traço reflete a tendência a experimentar angústia emocional e indica incapacidade de lidar com o estresse de forma eficaz. É ainda um fator de vulnerabilidade ao estresse bem conhecido, e robustamente associado com o início e o curso da depressão (Fanous, Neale, Aggen \& Kendler, 2007).

Koorevaar et al. (2013) encontraram que tanto a presença de um diagnóstico de depressão quanto seu nível de gravidade foram significativamente associados com maiores graus de neuroticismo, entre outros fatores de personalidade. É importante notar que as diferenças individuais na reação e percepção de estresse depois de experimentar eventos negativos da vida podem também depender de traços de personalidade.

Entre os vários fatores de vulnerabilidade ao estresse, níveis de Neuroticismo elevados foram longitudinalmente associados ao estresse episódico e a episódios depressivos (Kendler, Kuhn, \& Prescott, 2004). Segundo alguns autores, níveis mais elevados de Neuroticismo têm sido associados com aumento de sentimentos negativos e respostas comportamentais inadaptadas a experiências estressantes (Roesch et al., 2009), bem como a sintomas de ansiedade e depressão, apontando uma possível relação entre estresse e transtornos do humor (Uliaszek et al., 2010). Dessa forma, o estresse acumulado pode particularmente aumentar o risco de depressão em indivíduos com altos níveis de neuroticismo (Vinkers et al., 2014).

Conscienciosidade, por sua vez, é conhecida por estar associada à gestão do estresse e da tolerância (Besser $\&$ Shackelford, 2007). Segundo O'Connor, Conner, Jones, McMillan e Ferguson (2009), alta conscienciosidade parece estar associada a comportamentos de saúde adaptativos em resposta ao estresse cotidiano, levando indivíduos a responderem melhor ao estresse por se envolverem em mais comportamentos benéficos à saúde.

Acredita-se também que indivíduos com baixos níveis de conscienciosidade são menos propensos a usar enfrentamento focado no problema e, assim, são menos organizados no processo de atender às exigências da situação, e, como resultado, tornam-se mais vulneráveis às mudanças induzidas pelo estresse diariamente (O'Connor et al, 2009). Dessa forma, conscienciosidade pode moderar a relação entre a avaliação de problemas (como estressantes ou não) e o afeto positivo (Gartland, O'Connor, Lawton \& Ferguson, 2014).

No presente estudo, os sintomas de ansiedade e estresse associaram-se negativamente com os fatores de personalidade Extroversão e Abertura. Esses resultados indicam que indivíduos com mais ansiedade apresentam menores índices de extroversão e abertura, o que vai ao encontro da literatura, que indica que características pessoais contribuem muito para o desenvolvimento da ansiedade. Extroversão, por exemplo, é uma predisposição pessoal citada com frequência como uma das bases das reações emocionais, como a ansiedade (Marić, 2010).

Para Karsten et al. (2012), extroversão é um fator de predisposição também para o desenvolvimento de transtornos afetivos, como transtornos ansiosos. Inchausti, Delgado e Prieto (2015), por sua vez, encontraram baixa extroversão em pacientes com transtornos ansiosos, como TOC, por exemplo. Já segundo Uliaszek et al. (2010), um nível mais baixo de extroversão pode ser um dos fatores responsáveis pela ansiedade social, e esse traço de personalidade, entre outros, explicaria a associação entre o estresse e transtornos do humor.

No que tange à Abertura à experiência, Howard e Howard (1995) apontam que pessoas com baixos níveis desse traço de personalidade são, geralmente, mais rígidas e convencionais. Essa característica poderia levar ao desenvolvimento de sintomas ansiosos mediante experiências de diversidade e mudanças de planos repentinos.

Verificou-se ainda, neste estudo, que a autoeficácia foi a única variável preditora significativa tanto para o LOC interno quanto externo. A correlação entre LOC e autoeficácia já foi relatada na literatura (Baptista, Alves, \& Santos, 2008). Neste estudo, percebeu-se que autoeficácia 
foi capaz de predizer ambas as orientações de LOC, ou seja, uma alta autoeficácia é capaz de predizer maior LOC interno e menor LOC externo.

Maiores níveis de LOC externo relacionam-se à falta de cautela, o que pode resultar na falha em tomar medidas preventivas para evitar a ocorrência de desfechos desfavoráveis (Omizo \& Michael, 1982), que podem referir-se a desfechos profissionais, como a ocorrência de um acidente, por exemplo, ou desfechos pessoais, vinculados aos cuidados com a saúde. Dessa forma, indivíduos com alto LOC externo e baixa autoeficácia podem não sentir-se responsáveis pelos acontecimentos a sua volta e tão pouco capazes de modificar as circunstâncias dos mesmos. Por outro lado, indivíduos com altos índices de LOC interno deverão ser mais cuidadosos em suas atividades e experiências e responsáveis por elas, envolvendo-se menos em situações de risco. Um estudo desenvolvido com pilotos do exército encontrou que os participantes com menor LOC interno relataram significativamente mais acidentes recentes do que aqueles com alto LOC interno. Além disso, os autores concluíram que o LOC é uma variável relacionada ao envolvimento em acidentes e, dessa forma, demonstra sua importância, especialmente no contexto da aviação (Hunter \& Stewart, 2012).

Apesar de este estudo apresentar resultados importantes quando consideramos a formação e a prática de profissionais que atuam na área de aviação, é importante apontar algumas de suas limitações. Ressalta-se que o mesmo foi conduzido com uma amostra homogênea de acadêmicos, oriundos de apenas um curso de Ciências Aeronáuticas, o que pode dificultar a generalização dos resultados. As varáveis avaliadas enfocam prioritariamente aspectos de personalidade, que são importantes preditores comportamentais, mas que não esgotam a explicação dos determinantes da tomada de decisão em um contexto de prontidão de reposta. Nessa direção, sugere-se que pesquisas futuras investiguem a relação entre personalidade, a percepção de LOC e o desempenho cognitivo em tarefas experimentais de tomada de decisão em amostras de estudantes e em pilotos de aviação já atuantes.

Ainda assim, a partir dos resultados do presente estudo podem-se observar diversas correlações entre as variáveis analisadas: o LOC Interno correlacionou-se positivamente apenas com o fator de personalidade Conscienciosidade. A Autoeficácia correlacionou-se com todos os fatores de personalidade, sendo uma correlação negativa entre autoeficácia e neuroticismo e as demais positivas. Sintomas de Depressão correlacionaram-se positivamente com Neuroticismo e Amabilidade, e negativamente com Conscienciosidade. Já sintomas de Ansiedade e Estresse tiveram relações significativas e negativas com Abertura à Experiência, Extroversão e Conscienciosidade, mas positivas com Neuroticismo.

A avaliação de sintomas de estresse, de ansiedade e depressão e dos fatores de personalidade pode ser útil para o desenvolvimento de programas de intervenções básicas em nível de saúde, bem como podem auxiliar no processo seletivo destes indivíduos e no desenvolvimento de lideranças para esta classe profissional (Čabarkapa, Korica, \& Rodjenkov, 2011). Além disso, conhecer a orientação de LOC dos indivíduos em processos seletivos pode contribuir de forma relevante na segurança do voo e na diminuição do risco de acidentes.

No entanto, acredita-se que o principal resultado foi a predição significativa encontrada entre autoeficácia e LOC interno e externo. Esse resultado, especificamente, demonstrou que o LOC é uma variável importante a ser avaliada em estudantes e, especialmente, em pilotos de aeronáutica, pois pode predizer níveis altos ou baixos de autoeficácia - característica muito importante para esta profissão, uma vez que os pilotos, diariamente e com grande agilidade, devem tomar decisões importantes, além de gerenciar riscos e suas equipes.

\section{Referências}

Ambiel, R. A. M., Noronha, A. P. P., \& Nunes, M. F. O. (2012). Interesses profissionais e personalidade: um aporte para a integração dos construtos. Avaliação Psicológica, 11(2), 191-201.

Andrade, J. M. (2008). Evidências de Validade do Inventário dos Cinco Grandes Fatores de Personalidade para o Brasil. Tese de Doutorado não-publicada, Universidade de Brasília, Distrito Federal.

Bandura, A. (2012). On the functional properties of perceived self-efficacy revisited. Journal of Management, 38(9), 9-44. Bandura, A. (1994). Self-Efficacy. In V. S. Ramachaudran (Ed.), Encyclopedia of human behavior (4a ed., pp. 71-78). New York: Academic Press.

Baptista, M. N., Alves, G. A. D. S., \& Santos, T. M. D. M. D. (2008). Suporte familiar, autoeficácia e lócus de controle evidências de validade entre os construtos. Psicologia: ciência e profissão, 28(2), 260-271.

Benet-Martínez, V. \& John, O. P. (1998). Los cinco grandes across cultures and ethnic groups: multitrait multimethod analyses of the big five in Spain and English. Journal of Personality and Social Psychology, 75(3), 729-750. 
Besser, A., \& Shackelford, T. (2007). Mediation of the effects of the big five personality dimensions on negative mood and confirmed affective expectations by perceived situational stress: A quasi-field study of vacationers. Personality and Individual Differences, 42, 1333-1346.

Čabarkapa, M., Korica, V., \& Rodjenkov, S. (2011). Personal traits and a sense of job-related stress in a military aviation crew. Vojnosanit Pregl., 68(2), 143-149.

Caprara, G. V., Vecchione, M., Alessandri, G., Gerbino, M., \& Barbaranelli, G. (2011). The contribution of personality traits and self-efficacy beliefs to academic achievement: a longitudinal study. British Journal of Educational Psychology, 81, 78-96. doi: 10.1348/2044-8279.002004

Causse, M., Dehais, F., \& Pastor, J. (2011). Executive functions and pilot characteristics predict flight simulator performance in general aviation pilots. The International Journal of Aviation Psychology, 21(3), 217-234. doi: $10.1080 / 10508414.2011 .58244$

De Raad, B., \& Schouwenburg, H. C. (1996). Personality in learning and education: A review. European Journal of Personality, 10, 303-336.

Dolgin, D. L., Lambirth, T. T., Rentmeister-Bryant H. K., \& Moore, J. L. (2003). Selected personality characteristics of student naval aviators and student naval flight officers. International Journal of Aviation Psychology, 13(4), 415-427.

Fanous, A. H., Neale, M. C., Aggen, S. H., \& Kendler, K. S. (2007). A longitudinal study of personality and major depression in a population-based sample of male twins. Psychol Med, 37(8), 1163-1172.

Feijó, D., Câmara, V. M., \&, Luiz, R. R. (2014). Aspectos psicossociais do trabalho e transtornos mentais comuns em pilotos civis. Caderno de Saúde Pública, 30(11), 2433-2442. doi: 10.1590/0102-311X00151212

Fosse, T. H., Buch, R., Säfvenbom, R., \& Martinussen, M. (2015). The impact of personality and self-efficacy on academic and military performance: The mediating role of self-efficacy. Journal of Military Studies, 6(1), 47-65. doi: 10.1515/ jms-2016-0197

Galindo, O., \& Ardila, R. (2012). Psicología y pobreza. Papel del locus de control, la autoeficacia y la indefensión aprendida. Avances en Psicología Latinoamericana, 30(2), 381-407.

Ganesh, A., \& Joseph, C. (2005). Personality studies in aircrew: An overview. Indian Journal of Aerospace Medicine, $49(1), 54-62$

Gartland, N., O'Connor, D. B., Lawton, R., \& Ferguson, E. (2014). Investigating the effects of conscientiousness on daily stress, affect and physical symptom processes: A daily diary study. British Journal of Health Psychology, 19, 311-328.

Green, R. G., Muir, H., James, M., Gradwell, D., \& Green, R. L. (1996). Human Factors for Pilots. $2^{\mathrm{a}}$ ed. England: Avebury Aviation.

Hartman, R. O., \& Betz, N. E. (2007). The Five-Factor Model and Career Self-Efficacy: general and domain-specific relationships. J Career Assess., 15(2), 145-161. doi: 10.1177/1069072706298011.

Heckhausen, J., Wrosch, C., \& Schulz, R. (2010). A motivational theory of life-span development. Psychological Review, 117, 32-60. doi.org/10.1037/a0017668

Howard, P. J. \& Howard, J. M. (1995). The Big Five quick start: An introduction to the Five-Factor Model of Personality for human resource professionals. Charlotte, North Carolina: Center for Applied Cognitive Studies. Recuperado em 11 setembro 2016, de http://www.eric.ed.gov/ERICWebPortal/search/detailmini.jsp?_nfpb=true\&_\& ERICExtSearch_SearchValue_0=ED384754\&ERICExtSearch_SearchType_0=no\&accno=ED384754.

Hunter, D. R. (2002). Development of an aviation safety locus of control scale. Aviation, Space, and Environmental Medicine, 73, 1184-1188.

Hunter, D. R., \& Stewart, J. E. (2012). Safety locus of control and accident involvement among army aviators. The International Journal of Aviation Psychology, 22(2), 144-163. doi: 10.1080/10508414.2012.663244

Hurtz, G. M., \& Donovan, J. J. (2000). Personality and job performance: The Big 5 revisited. Journal of Applied Psychology, 85, 869-879.

Inchausti, F., Delgado, A. R., \& Prieto, G. (2015). Obsessive-compulsive disorder and its relationship with disgust vulnerability and conscientiousness. Psicothema, 27(3), 254-260. doi: 10.7334/psicothema2015.7

Infurna, F. J., \& Okun, M. A. (2015). Antecedents and outcomes of level and rates of change in perceived control: The moderating role of age. Developmental psychology, 51(10), 1420-1437. doi.org/10.1037/a0039530

Infurna, F. J., Ram, N., \& Gerstorf, D. (2013). Level and change in perceived control predict 19-year mortality: Findings from the Americans' Changing Lives Study. Developmental Psychology, 49, 1833-1847. doi.org/10.1037/ a0031041 
Itani, A. (2009). Saúde e gestão na aviação: a experiência de pilotos e controladores de tráfego aéreo. Psicologia e Sociedade, 21, 203-12. doi:10.1590/S01021822009000200007

Judge, T. A., \& Ilies, R. (2002). Relationship of Personality to Peformance Motivation. Journal of Applied Psychology, 87(4), 797-807. doi: 10.1037//0021-9010.87.4.793

Karsten, J., Penninx, B. W., Riese, H., Ormel, J., Nolen, W. A., \& Hartman, C. A. (2012). The state effect of depressive and anxiety disorders on big five personality traits. J Psychiatr Res, 46(5), 644-50. doi: 10.1016/j.jpsychires.2012.01.024.

Kendler, K. S., Kuhn, J., \& Prescott, C. A. (2004). The interrelationship of neuroticism, sex, and stressful life events in the prediction of episodes of major depression. The American Journal of Psychiatry, 161(4), 631-636.

Kim, S. E., Kim, H., Cho, J., Kwon, M., Chang, Y., ... Kim, H. (2016). Direct and Indirect Effects of Five Factor Personality and Gender on Depressive Symptoms Mediated by Perceived Stress. Plos One, 11(6), e0157204. doi: 10.1371/ journal.pone. 0157204

Koorevaar, A. M., Comijs, H. C., Dhondt, A. D., van Marwijk, H. W., van der Mast, R. C., ... Stek, M.L. (2013). Big Five personality and depression diagnosis, severity and age of onset in older adults. J Affect Disord., 151(1), 178-85. doi: 10.1016/j.jad.2013.05.075 PMID: 23820093.

Lazarus, R. S., \& Folkman, S. (1984). Stress, appraisal, and coping. New York: Springer

Lent, R., Brown, S., \& Hackett, G. (1994). Toward a unifying social cognitive theory of career and academic interest, choice and performance. Journal of Vocational Behavior, 45, 79-122.

Lovibond, S. H., \& Lovibond, P. F. (1995). Manual for the Depression Anxiety Stress Scales. Sydney: Psychology Foundation.

Mali, V. (2013). A Study on Locus of Control and its Impact on Employees' Performance. International Journal of Science and Research (IJSR), 2(12), 149-151.

Marić, M. (2010). Personality traits, life events and adolescent anxiety. Psychology Applied, 19(1), 39-57.

Noronha, A. P. P., Mansão, C. S. M., \& Nunes, M. F. O. (2012). Interesses Profissionais e Personalidade: análise correlacional a partir do ATPH e BFP. Actualidades en Psicología, 26(113), 73-86.

Nunes, C. H. S., \& Hutz, C. S. (2007). Escala Fatorial de Socialização: manual técnico. São Paulo: Casa do Psicólogo.

Nunes, C. H. S., Hutz, C. S., \& Giacomoni, C. H. (2009). Associação entre bem-estar subjetivo e personalidade no modelo dos cinco grandes fatores. Avaliação Psicológica, 8(1), 99-108.

Nunes, C. H. S., Hutz, C. S., \& Nunes, M. F. O. (2010). Bateria Fatorial de Personalidade: manual técnico. São Paulo: Casa do Psicólogo.

O’Connor, D.B., Conner, M., Jones, F., McMillan, B., \& Ferguson, E. (2009). Exploring the benefits of conscientiousness: an investigation of the role of daily stressors and health behaviors. Ann Behav Med., 37(2), 184-96. doi: 10.1007/ s12160-009-9087-6

Omizo, M. B., \& Michael, W. B. (1982). Biofeedback-induced relaxation training and impulsivity, attention to task, and locus of control among hyperactive boys. Journal of Learning Disabilities, 15, 414-416.

Pienaar, J., \& De Witte, H. (2016). Work locus of control and sense of coherence as antecedents of job insecurity. African Journal of Business Management, 47(3), 35-43. doi: 10520/EJC194109

Poropat, A. E. (2009). A meta-analysis of the five-factor model of personality and academic performance. Psychol Bull., 135(2), 322-38. doi: 10.1037/a0014996.

Roesch, S. C., Aldridge, A. A., Vickers, R. R. Jr., \& Helvig, L. K. (2009). Testing personality-coping diatheses for negative and positive affect: a longitudinal evaluation. Anxiety, stress, and coping, 22(3), 263-81. doi: 10.1080/10615800802158419

Ruthig, J. C., Perry, R. P., Hladkyj, S., Hall, N. C., Pekrun, R., \& Chipperfield, J. G. (2008). Perceived control and emotions: interactive effects on performance in achievement settings. Soc Psychol Educ., 11, 161-180. doi 10.1007/ s11218-007-9040-0

Schwarzer, R., \& Jerusalem, M. (1995). Generalized Self-Efficacy Scale. In J. Weinman, S. Wright \& M. Johnston (Eds.). (pp. 35-37). Measures in health psychology: a user's portfolio. Causal and control beliefs. Windsor. Uk: Nfer-Nelson.

Shell, D. F., \& Husman, J. (2001). The Multivariate Dimensionality of Personal Control and Future Time Perspective Beliefs in Achievement and Self-Regulation. Contemp Educ Psychol., 26(4), 481-506.

Uliaszek, A. A., Zinbarg, R. E., Mineka, S., Craske, M. G., Sutton, J. M., ... Hammen, C. (2010). The role of neuroticism and extraversion in the stress-anxiety and stress-depression relationships. Anxiety, stress, and coping, 23(4), 363-81. doi: 10.1080/10615800903377264 
Vinkers, C. H., Joels, M., Milaneschi, Y., Kahn, R. S., Penninx, B. W. J. H., \& Boks, M. P. M. (2014). Stress exposure across the life span cumulatively increases depression risk and is moderated by neuroticism. Depression and Anxiety, 31, 737-745.

Wang, Y., Yao, L., Li, L., Yang, X., Wu, H., ... Wang, L. (2014). The mediating role of self-efficacy in the relationship between Big Five personality and depressive symptoms among Chinese unemployed population: a cross-sectional study. BMC Psychiatry, 14(1), 61.

Widyahening, I. S. (2007). High level of work stressors increase the risk of mental-emotional disturbances among airline pilots. Medical Journal of Indonesia, 16(2), 117-21. doi: 10.13181/mji.v16i2.267

You, X., Ji, M., \& Han, H. (2013). The effects of risk perception and flight experience on airline pilots' locus of control with regard to safety operation behaviors. Accident Analysis and Prevention, 57, 131-139.

Recebido 11/08/2017

Aceite Final 25/09/2018

Sobre os autores

Manuela Polidoro Lima é psicóloga, mestre em Ciências da Saúde, com ênfase em Oncologia, doutora em Psicologia Clínica pelo Programa de Pós-Graduação em Psicologia da Pontifícia Universidade Católica do Rio Grande do Sul (PUCRS).

Luiza Signorelli é psicóloga, graduada pela PUCRS. Bolsista de Iniciação Científica de 2015 até 2017.

Maria Laura Del Huerto é psicóloga, graduada pela PUCRS. Estagiária em Psicologia Clínica no Serviço de Atendimento e Pesquisa em Psicologia da PUCRS (SAPP). Bolsista de Iniciação Científica de 2015 até 2017.

Thiago Gomes de Castro é psicólogo, mestre e doutor em Psicologia pela Universidade Federal do Rio Grande do Sul (UFRGS). Atualmente é professor adjunto no Instituto de Psicologia da UFRGS.

Manoela Ziebell de Oliveira é psicóloga, mestre e doutora em Psicologia pela Universidade Federal do Rio Grande do Sul. Atualmente é Professora do Programa de Pós-Graduação em Psicologia da Escola de Ciências da Saúde da PUCRS e responsável pelo Grupo de Estudos em Desenvolvimento de Carreira da PUCRS.

Tatiana Quarti Irigaray é psicóloga, possui especialização em Psicologia Clínica com ênfase em Avaliação Psicológica e Neuropsicologia pela UFRGS. Doutora e mestre em Gerontologia Biomédica pela Pontifícia Universidade Católica do Rio Grande do Sul (PUCRS). Possui pós-doutorado em Psicologia pela PUCRS. Atualmente é Professora e Coordenadora do Grupo de pesquisa Avaliação, Reabilitação e Interação Humano-Animal, do Programa de PósGraduação em Psicologia da Escola de Ciências da Saúde da PUCRS. 